\title{
Multiple breakpoint method for measuring effect of antibiotics on endocarditis strains of streptococci
}

\author{
J Marks, A Paull
}

\begin{abstract}
The activity of penicillin alone and combined with aminoglycoside on endocarditis strains of streptococci was examined. Good assay reproducibility was obtained by the use of logarithmic phase cultures standardised by opacity, careful inoculation of well-plates, removal of antibiotic by membrane transfer and incubating survival counts in hydrogen plus carbon dioxide. The use of 10-fold intervals for penicillin concentration simplified assay design without loss of efficiency.
\end{abstract}

The wide variety of methods used to measure the effect of antibiotics on streptococci suggests that none of them merits general acceptance. $^{12}$ There are also major disagreements in interpretation. In the United States cultures in the logarithmic phase of growth are recommended for the determination of tolerance to penicillin; in Britain most laboratories use the stationary phase which is much less sensitive ${ }^{3}$ (Snell JJS, Central Public Health Laboratory; personal communication). We describe a method which permits a compromise between these two standpoints because its reproducibility is good enough to allow two degrees of tolerance to be distinguished. Bacteriostatic action, which is of minor importance, is expressed by the "breakpoint MIC", the penicillin concentrations being set at 10 -fold intervals. The bactericidal action of penicillin both alone and combined with aminoglycoside is estimated by survival counts using the penicillin at 10 breakpoint minimum inhibitory concentration (MIC). This concentration lies between $10 \mathrm{MIC}$ and $50 \mathrm{MIC}$ and was found in a preliminary study to define tolerance in exactly the same way as the commonly used level of 32 MIC. Inocula are used in the logarithmic phase of growth and standardised by opacity, giving results shown to be more reproducible than those obtained with cultures in the stationary phase and dependent on initial viable counts. Our method is not designed specifically to show antibiotic synergy, although this often becomes evident. On the assumption that a combination of penicillin and aminoglycoside is the regimen of first choice, its aim is to determine whether such a pairing is likely to be highly bactericidal in practice, to choose between different aminoglycosides, and to suggest how intensively the latter should be used, bearing in mind their potential toxicity.
Only gentamicin and netilmicin, however, are considered here.

\section{Methods}

Seventeen unselected endocarditis strains of viridans streptococci and two of Enterococcus faecium were studied. To extend our experience of enterococci, the latter were supplemented with eight non-speciated strains isolated from other types of clinical infection.

The liquid medium was digest broth containing $0.1 \%$ glucose (Southern Group Laboratories) with the addition of $2.5 \%$ horse serum. The serum was necessary for the adequate growth of some strains but harmful in higher concentrations to others. Broth was distributed in $3 \mathrm{ml}$ volumes in $7.5 \mathrm{ml}$ plastic bottles (Sterilin) and incubated for sterility. Solid medium was standard dried horse blood agar.

A well dispersed culture of streptococci suspended in $1 \%$ formalin was matched to McFarland standards 4,2 , and 0.5 for distribution into $7.5 \mathrm{ml}$ plastic bottles. No change was apparent during storage for a year at $4^{\circ} \mathrm{C}$.

A heavy subculture on blood agar was incubated for 24 hours at $37^{\circ} \mathrm{C}$ in hydrogen plus $5-10 \%$ carbon dioxide (BBL Gaspak). The film of fluid in a $2 \mathrm{~mm}$ wire loop dipped in broth was charged by dragging it through the growth and used to inoculate $3 \mathrm{ml}$ of prewarmed broth. The subculture was vortexmixed for five seconds and if necessary diluted to bring its opacity just below that of the 0.5 standard. Incubation in air at $37^{\circ} \mathrm{C}$ followed until the opacity reached that of standard 4, usually taking two to four hours. The culture was then vortex-mixed for 30 seconds, diluted with sterile distilled water to match standard 2 (chosen for ease of matching) and finally diluted again 1 in 5 for use. Meanwhile control broth and broths containing sodium benzyl penicillin to give final concentrations of $0 \cdot 01,0 \cdot 1,1$ and $10 \mathrm{mg} / 1$ were distributed in $340 \mu \mathrm{l}$ volumes in a row of a microwell plate (Nunc $96 \mathrm{~F}$ ). Two similar rows were set up to include final concentrations of $2 \mathrm{mg} / 1$ gentamicin and $4 \mathrm{mg} / 1$ netilmicin, respectively. Bubbles in broth were removed with a hot wire.

A micropipette with a range to $250 \mu \mathrm{l}$ was used to distribute medium and one ranging to $100 \mu \mathrm{l}$ for inoculation and sampling. A standardised and diluted culture $(20 \mu \mathrm{l})$ was inoculated into each well, introducing and 
withdrawing the tip quietly and vertically at the centre of the broth and discharging the inoculum into it gently without mixing, precautions taken to prevent leaving organisms above the broth meniscus. The wellplate was kept level at all times and not agitated. A separate suspension and tip were used for each row to prevent unwanted transfer of antibiotic. The titration was incubated in air at $37^{\circ} \mathrm{C}$ for 24 hours. The breakpoint MIC for penicillin was read in the first row. The three broths containing 10 breakpoint MIC of penicillin (alone and with each aminoglycoside) were well mixed by micropipette and sampled. When the penicillin breakpoint $\mathrm{MIC}$ was $\geqslant 10 \mathrm{mg} / \mathrm{l}$, each visibly clear broth at the $10 \mathrm{mg} / \mathrm{l}$ level was sampled.

Samples for survival counts were two volumes of $20 \mu \mathrm{l}$, each spread over an area of about $150 \mathrm{~mm}^{2}$ on a cellulose acetate-nitrate filter membrane (Millipore, GS $0.22 \mu \mathrm{m}, 47$ $\mathrm{mm}$ diameter), which had been laid aseptically, shiny side up, on a blood agar plate. Dilutions were also plated for the present study but are not required in ordinary practice. After incubation for one hour at $37^{\circ} \mathrm{C}$ the membrane was transferred to a second blood agar plate for a second hour's incubation and then to a third plate for final incubation (now with the plate facing downwards) in hydrogen plus carbon dioxide at $37^{\circ} \mathrm{C}$. This method of removing free antibiotic is a slight modification of that previously described. ${ }^{4}$ A preliminary reading was made after 24 hours in case of overcrowding by eugonic colonies and a final reading at 48 hours using a $\times 5$ hand lens with the plate held obliquely to show tiny colonies more effectively. It is not permissible to reduce the incubation time or to incubate in either air or air plus carbon dioxide. ${ }^{5}$

Broth cultures were incubated in air at $37^{\circ} \mathrm{C}$ for 18 hours. Viable counts were made on blood agar for incubation in hydrogen plus carbon dioxide at $37^{\circ} \mathrm{C}$. They could be read at 24 hours (not having been exposed to antibiotic) but the counts were not predictable. Tests laid out as described above were therefore made in triplicate using the 18 hour cultures undiluted and at 1 in 5 and 1 in 25 dilutions. The set that proved to have an initial inoculum exceeding but nearest to $5 \times$ $10^{5} / \mathrm{ml}$ in its reaction mixtures was chosen for reading and sampling. Survival counts were made as described above and expressed as percentage kill. Enterococci were not tested in the stationary phase of culture.

Tests were repeated at intervals of at least a week. As only quantitation was being studied, aliquots of a single batch of medium were used and relatively few batches of antibiotic. The potency of the latter was checked by titration with the Oxford staphylococcus. The possible effects of medium variation are considered below.

\section{Results}

VIRIDANS STREPTOCOCCI

The penicillin breakpoint MIC for strain 9

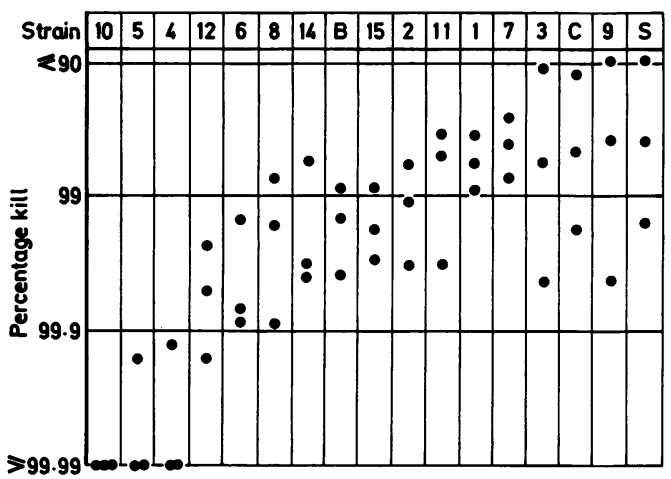

Figure 1 Percentage kill at 24 hours by penicillin at 10 breakpoint MIC on stationary phase cultures of 17 endocarditis strains of viridans streptococci: results of three separate tests.

was $0.01 \mathrm{mg} / 1$ and for the other 16 strains it was $0.1 \mathrm{mg} / \mathrm{l}$. Survival counts after exposure to 10 breakpoint MIC in three examinations of stationary phase cultures are presented in fig 1 . On the criterion of a $99.9 \%$ kill, all but strains 10,5 , and 4 were tolerant. There was only a single aberrant reading of tolerance in the 51 tests (strain 12), but the scatter of the replicate tests was too great to allow different degrees of tolerance to be distinguished with confidence.

The response of logarithmic phase cultures to penicillin alone and in combination with either gentamicin or netilmicin is presented in fig 2 . The reproducibility of repeated tests was clearly better than that seen in tests during the stationary phase. Initial counts are not required for the log phase method but in preliminary studies the inoculum chosen was shown to give a mean count of $5 \times 10^{6} \mathrm{cfu} / \mathrm{ml}$ in reaction mixtures while a survival count of $20 \mathrm{cfu}$ corresponded to about a $99.9 \%$ kill of stationary phase cultures in distinguishing tolerance to penicillin. To allow a safety margin for Poisson distribution, however, a line has been drawn 2 SD below this at $10 \mathrm{cfu}$ of survival. Another has been drawn at $100 \mathrm{cfu}$ of survival which appears to distinguish reasonably well a moderate from a poor degree of bactericidal activity. The reproducibility of the survival counts obtained with combina-

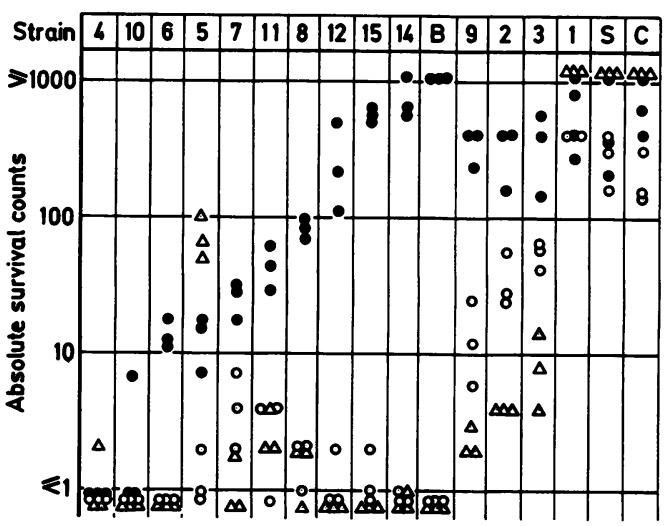

Figure 2 Absolute survival counts of log phase cultures of 17 endocarditis strains of viridans streptococci, standardised by opacity, after exposure for 24 hours to penicillin at 10 breakpoint MIC either alone (O), in combination with $2 \mathrm{mg} / \mathrm{l}$ gentamicin ( $O$ ), or with $4 \mathrm{mg} / \mathrm{l}$ netilmicin $(\triangle)$ : results of three separate tests. 
tions of penicillin and aminoglycoside was also satisfactory. Their interpretation will be considered below but it may be noted here that netilmicin seemed to be a more effective partner to penicillin than gentamicin was in the case of strains 9,2 , and 3 and less effective with strain 5 . Netilmicin was antagonistic to penicillin with strains $5,1, \mathrm{~S}$ and $\mathrm{C}$.

\section{ENTEROCOCCI}

The penicillin breakpoint MIC was $10 \mathrm{mg} / 1$ for nine of the strains in $\log$ phase tests and 1 $\mathrm{mg} / \mathrm{l}$ for endocarditis strain $16 \mathrm{E}$. Because of the relative insensitivity of enterococci to penicillin only its combinations at $10 \mathrm{mg} / 1$ with gentamicin and netilmicin will be considered. Both combinations were highly bactericidal for eight strains (survival counts of $\leqslant 10$ ). Penicillin was highly bactericidal in combination with gentamicin for strain $16 \mathrm{E}$ but only moderately so with netilmicin (survival counts between 10 and 100). It was only moderately bactericidal with either aminoglycoside in the case of one non-endocarditis strain.

\section{Discussion}

The use of multiple breakpoints for penicillin simplifies the format of tests in the present field but the main advantage of the method described is good reproducibility. The most important contributory factor is its use of logarithmic phase cultures standardised by opacity which avoids the problems introduced by variations in chain length, clumping, and over-maturity associated with stationary phase cultures. The recommended well-plates have wide apertures, allowing clean entry of inocula by micropipette, which, with avoidance of shaking, minimises the risk of depositing organisms where they may escape exposure to antibiotic. Other features are the use of membrane transfer to remove antibiotic efficiently, permitting the use of large samples, and the incubation of membranes in an atmosphere of hydrogen plus carbon dioxide for $\mathbf{4 8}$ hours which favours the demonstration of survival. ${ }^{5}$

Opinions may differ as to the interpretation of the results presented but our own suggestions are as follows. Bacteriostatic activity can be expressed as breakpoint MIC, but reporting it to clinicians serves no purpose and may cause confusion. The convention recommended for reporting the activity of penicillin and of its combination with aminoglycosides is "highly bactericidal" when the survival count is $\leqslant 10$, "moderately bactericidal" when it lies between 10 and 100, and "poorly bactericidal" when it exceeds 100 . On this basis penicillin would be reported as highly bactericidal alone for strains 10 and 4 and the addition of aminoglycoside to the treatment regimen need be only minimal. It would be reported as moderately bactericidal for strains $6,5,7,11$ and 8 , but its combination with either gentamicin or netilmicin as highly bac- tericidal except in the case of strain 5 where netilmicin was antagonistic to penicillin. Penicillin alone was poorly bactericidal to strains $12,15,14$ and $B$ but its combination with either aminoglycoside was highly bactericidal. In the case of the last two groups the recommendation would be a standard regimen of penicillin plus either aminoglycoside, excluding netilmicin for strain 5 . With strains 9,2 , and 3 penicillin alone was poorly bactericidal but it was moderately bactericidal in combination with gentamicin and highly bactericidal with netilmicin, the latter combination obviously being preferable. In the case of these three strains the netilmicin should perhaps be used at the upper end of the tolerable dosage range. Penicillin was poorly bactericidal both alone and in combination in tests on strains $1, S$, and $C$ and an alternative form of treatment would be indicated for these infections.

Strains with survival counts between 10 and $100 \mathrm{cfu}$ would usually be considered to be tolerant in Britain and non-tolerant in the United States, but are here judged to be intermediate. The present experience suggests that such a compromise will seldom be necessary for a combination of penicillin with the best available aminoglycoside.

Bactericidal activity is very susceptible to changes in test conditions. Any deviation from those described above would require confirmation of the 10 and $100 \mathrm{cfu}$ parameters, or more likely, the definition of new ones. It is standard practice to confirm the potency of the antibiotics used in bactericidal assays but not always to test the medium used. Each batch of medium should be checked with strains of known behaviour, and if possible one with an intermediate degree of tolerance should be run as a control to the unknown strain. Such precautions, together with the care needed to get accurate results in tests of bactericidal activity, make it desirable for these to be entrusted to laboratories with a special interest in the subject, if not for the initial examination, then for confirmation purposes.

We thank colleagues in the Welsh Region and Dr G Colman, Central Public Health Laboratory, Colindale, for contributing to the strains in our study.

1 Stratton CW. The role of the microbiology laboratory in the treatment of infective endocarditis. $J$ Antimicrob Chemother 1987;20 (Suppl A):41-9.

2 Eykyn SJ. The role of the laboratory in assisting treatmenta review of current UK practices. J Antimicrob Chemother 1987;20 (Suppl A):51-64.

3 National Committee for Clinical Laboratory Standards. Methods for determining bactericidal activity of antimicrobial agents 1987;7:35-74.

4 Paull A, Marks J. A new method for the determination of bactericidal antibiotic synergy. $J$ Antimicrob Chemother

5 Marks J, Paull A. Effect of penicillin on endocarditis strains of viridans streptococci. J Clin Pathol 1989;42:1114-5. 